\title{
Numerical simulation on local scour below a weir using Two-phase WC-SPH method
}

\author{
Ahmed M. Abdelrazek \\ Hydraulic Research Laboratory, Graduate School of Engineering, Hokkaido University, Hokkaido, Japan
}

Irrigation \& Hydraulic Department, Faculty of Engineering, Alexandria University, Alexandria, Egypt

I. Kimura \& Y. Shimizu

Hydraulic Research Laboratory, Graduate School of Engineering, Hokkaido University, Japan

\begin{abstract}
In this study, numerical simulation of the local scour behind a weir due to overflow using a Lagrangian formulation of the Navier-Stokes equations, based on the weakly compressible smoothed particle hydrodynamics (WC-SPH) method, has been done. In this simulation, the advantages of SPH will be exploited to simulate the soil-water interaction. Water is considered as a viscous fluid with weak compressibility and soil is assumed to be an elastic-plastic material. The elastic-perfectly plastic model based on MohrCoulomb's failure criterion is implemented in SPH formulations to model the soil movement. Interaction between soil and water is taken into account by means of seepage force and pore water pressure. Numerical Simulation of local scour behind the weir has been done; the numerical results are then compared with experimental data. The results have shown that the proposed model could be considered a powerful tool to simulate extremely large deformation and failure of soil.
\end{abstract}

\section{INTRODUCTION}

The interaction between water and soil poses problems in different areas of marine, Geomechanics and hydraulic engineering. Numerical predictions for such interactions in the case of large deformations could provide useful knowledge for engineering practice and design.

There are some traditional numerical methods used for simulating the deformation and failure of geometries in the framework of continuum mechanics, such as finite element method, finite difference method and boundary element method. These methods have been successfully implemented. On the other hand, in the case of large deformation problems, the previous methods produce instabilities due to excessive distortion of a mesh.

Recently a new class of numerical methods, which are called mesh-free methods, has been developed. Mesh-free methods do not require Eulerian grids and they deal with a number of particles in a Lagrangian framework. Many mesh-free methods have been developed in the last decades, among these methods; the Smoothed Particle Hydrodynamics $(\mathrm{SPH})$ method will be exploited to simulate the soil-water interaction.

SPH method is a mesh-free particle method and it is considered to be one of the most modern meshfree particle techniques. It was originally invented for astrophysical applications; then it has been applied in a huge range of applications such as, free surface fluid flow (Monaghan, 1994) and (Abdelrazek et al. 2014), multi-phase flow (Monaghan \& Kocharyan, 1995), snow avalanching (Abdelrazek et al. 2014), and gravity granular rapid flow (Shinichi et al. 2011, Abdelrazek et al. 2015, 2016). In SPH method, each particle in the domain carries all field variable information such as density, pressure, velocity and it moves with the material velocity. The governing equations in the form of partial differential equations are converted to the particle equations of motion, and then they are solved by a suitable numerical scheme.

In this simulation, the advantages of SPH will be exploited to simulate the soil-water interaction. Water is considered as a viscous fluid with week compressibility and soil is assumed to be an elasticplastic material. The elastic-perfectly plastic model based on Mohr-Coulomb's failure criterion is implemented in SPH formulations to model the soil movement (Bui et al. 2007 \& 2010).

Modeling of soil and water in the framework of the smoothed particle hydrodynamics method have been presented and verified as one-phase flow models in the authors' former published papers (Abdelrazek et al. 2014, 2015, 2016), however, these models only allow to simulate soil-water interaction.

The interaction between soil and water is taken into account by means of seepage force and pore water pressure in order to conduct the two-phase model. 
The aim of this study is to test the predictive power of the WCSPH method to simulate local scour, which consider a typical for the natural waterfall, also be observed in the plunging jet pool downstream of hydraulic structures, such as a weir in a river or the spillway of a dam.

\section{SPH FORMULATION}

The SPH method is a continuum-scale numerical method. The material properties $f(x)$, at any point $\mathrm{x}$ in the simulation domain are calculated according to an interpolation theory over its neighboring particles which are within its influence domain $\boldsymbol{\Omega}$ as shown in figure1, through the following formula,

$$
\langle f(x)\rangle=\int_{\Omega} f\left(x^{\prime}\right) W\left(x-x^{\prime}, h\right) d x^{\prime}
$$

where $h$ is the smoothing length defining the inflence domain of the kernel estimate and $W\left(x-x^{\prime}, h\right)$ is the smoothing function, which must satisfy three conditions (Liu \& Liu 2003): the first condition is the normalization,

$\int_{\Omega} W\left(x-x^{\prime}, h\right) d x^{\prime}=1$

the second one is the Delta function condition,

$$
\lim _{h \rightarrow 0} W\left(x-x^{\prime}, h\right)=\delta\left(x-x^{\prime}\right)
$$

and the third condition is the compact condition,

$W\left(x-x^{\prime}, h\right)=0 \quad$ when $\left|x-x^{\prime}\right|>k h$

where $k$ is a constant depending on the type of smoothing function.

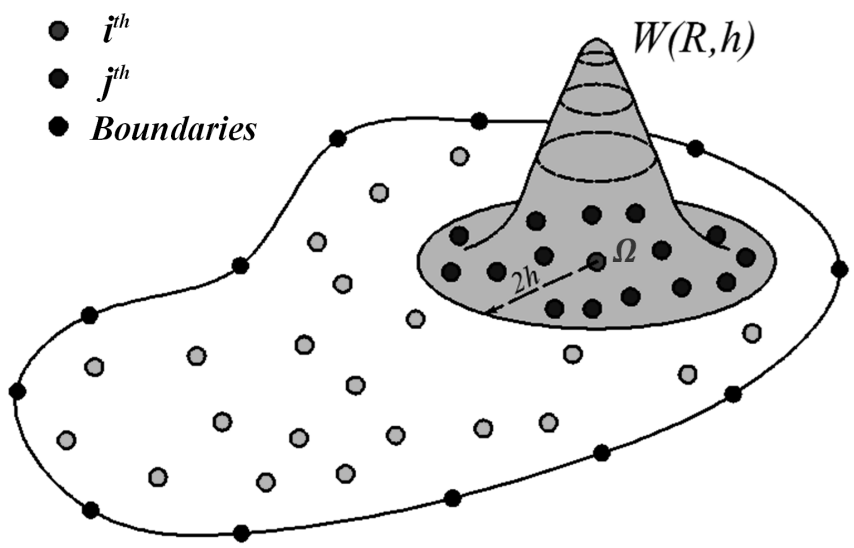

Figure 1. Particle approximation based on kernel function $W$ in influence domain $\boldsymbol{\Omega}$ with radius $k h(k=2)$

There are many possible types of smoothing functions, which can satisfy the aforementioned condi- tions. The most known function, among them, is the cubic spline interpolation function, and Wendland function. In this study, we used the cubic spline interpolation function which was proposed by Monaghan and Lattanzio (1985), and it is defined as:

$$
\begin{aligned}
& W(R, h)= \\
& \alpha_{d} \times\left\{\begin{array}{cl}
1.5-R^{2}+0.5 R^{3} & 0 \leq R<1 \\
(2-R)^{3} / 6 & 1 \leq R<2 \\
0 & R \geq 2
\end{array}\right.
\end{aligned}
$$

where $\alpha_{d}=\frac{3}{2 \pi h^{3}}$ in $3 \mathrm{D}$ space, and $R=\frac{\left(x-x^{\prime}\right)}{h}$.

In the SPH method, the calculation domain is represented by a finite number of particles, which carry mass and the field variable information such as density, stress, etc. (Liu \& Liu 2003, 2010). Accordingly, the continuous integral representation for $f(x)$ is approximated in the following form:

$$
\begin{aligned}
\langle f(x)\rangle=\int_{\Omega} f\left(x^{\prime}\right) W\left(x-x^{\prime}, h\right) d x^{\prime} & \\
& \approx \sum_{j=1}^{N} f\left(x_{j}\right) W\left(x-x^{\prime}, h\right) \frac{m_{j}}{\rho_{j}}
\end{aligned}
$$

Using equation (6), the approximation function of each particle $i$ can be written as,

$\left\langle f\left(x_{i}\right)\right\rangle=\sum_{j=1}^{N} \frac{m_{j}}{\rho_{j}} f\left(x_{j}\right) W_{i j}$

where $W_{i j}=W\left(x-x^{\prime}, h\right)$

Equation (7) states that the value of the function at particle $i$ is approximated using a weighted average of those values of the function at all other particles in the influence domain of particle $i$. Following the same argument, the particle approximation of the spatial derivative of a function at any particle $i$ is,

$$
\left\langle\nabla . f\left(x_{i}\right)\right\rangle=\sum_{j=1}^{N} \frac{m_{j}}{\rho_{j}} f\left(x_{j}\right) \nabla_{i} W_{i j}
$$

\section{SPH MODELS FOR WATER AND SOIL}

For a fluid like water, it is customary to model it as exactly incompressible. However, the approach in $\mathrm{SPH}$ is different; the real fluid is approximated by an 
artificial fluid which is more compressible than the real one. The governing equations for fluid flow are the well-known Navier-Stokes equations, which in the Lagrangian description state the conservation of continuity and momentum as follows:

$$
\begin{gathered}
\frac{D \rho}{D t}=-\rho \frac{\partial v^{\alpha}}{\partial x^{\alpha}} \\
\frac{D v^{\alpha}}{D t}=\frac{1}{\rho}\left(\frac{\partial \sigma^{\alpha \beta}}{\partial x^{\alpha}}\right)+f^{\alpha}
\end{gathered}
$$

where $\rho$ is the density; $v$ is velocity; $\sigma^{\alpha \beta}$ is stress tensor, $f^{\alpha}$ is the component of acceleration caused by external force.

The stress tensor, $\sigma^{\alpha \beta}$, normally consists of two parts: an isotropic pressure $P$ and a viscous shear stress, which is proportional to the shear strain rate denoted by $\varepsilon$ through the viscosity $\mu$,

$\sigma^{\alpha \beta}=-P \delta^{\alpha \beta}+\mu \varepsilon^{\alpha \beta}$

where:

$\varepsilon^{\alpha \beta}=\frac{\partial v^{\alpha}}{\partial x^{\beta}}+\frac{\partial v^{\beta}}{\partial x^{\alpha}}-\frac{2}{3}\left(\frac{\partial v^{\gamma}}{\partial x^{\gamma}}\right) \delta^{\alpha \beta}$

Equation of state is used to estimate the pressure change of water

$P=B\left[\left(\frac{\rho}{\rho_{o}}\right)^{\lambda}-1\right]$

where $\lambda$ is a constant $(=7), \rho_{o}$ is the reference density, $B$ is a problem dependent parameter, which sets a limit for the maximum change of the density and will be calculated as

$B=\frac{100 V_{\text {type }}^{2} \rho_{o}}{\lambda}, V_{\text {type }}=\sqrt{2 \mathrm{~g} H}$

Modeling the behavior of soil using the SPH method is similar to that of water. The SPH form of conservation equations (9) and (10) are still used to estimate the density and motion of soil particles. The key difference between these two models is the calculation of the stress tensor appearing in Eq. (2), in which the pressure and stress-strain relationship of soil are calculated differently from those of water; soil is assumed herein to be an elastic-plastic material. The stress tensor of soil is made up of two parts: isotropic pressure $P$ and deviatoric shear stress $S$,

$\sigma^{\alpha \beta}=-P \delta^{\alpha \beta}+S^{\alpha \beta}$

Since soil is assumed to have elastic behavior (Bui and others, 2007; Yaidel and others 2012), so the pressure equation of soil will obey Hooke's law, as follows,
$P=-K \frac{\Delta V}{V}=K\left(\frac{\rho}{\rho_{o}}-1\right)$

where $K$ is bulk modulus; $\Delta V / V$ is the volumetric strain; and $\rho_{o}$ is the initial density of soil. The rate of change of deviatoric shear stress $d S / d t$ can be calculated using shear modulus, $\mu$, using the Jaumann rate from the following constitutive equation,

$\frac{d S^{\alpha \beta}}{d t}=2 \mu\left(\dot{\varepsilon}^{\alpha \beta}-\frac{1}{3} \dot{\varepsilon}^{\gamma \gamma}\right)+S^{\alpha \gamma} \omega^{\beta \gamma}$
$+\omega^{\gamma \beta} S^{\alpha \gamma}$

where $\dot{\varepsilon}^{\gamma \gamma}=\dot{\varepsilon}^{x x}+\dot{\varepsilon}^{y y}+\dot{\varepsilon}^{z z}$, is the strain rate tensor and $\omega^{\gamma \beta}$ is the rotation rate tensor. It can be defined by,

$\dot{\varepsilon}^{\alpha \beta}=\frac{1}{2}\left(\frac{\partial v^{\alpha}}{\partial x^{\beta}}+\frac{\partial v^{\beta}}{\partial x^{\alpha}}\right)$

$\omega^{\alpha \beta}=\frac{1}{2}\left(\frac{\partial v^{\alpha}}{\partial x^{\beta}}-\frac{\partial v^{\beta}}{\partial x^{\alpha}}\right)$

Using the concept of the SPH approximation the system of partial differential equations (1) and (2) can be converted into the SPH formulations which will be used to solve the motion of soil particles as follows:

$\frac{D \rho_{i}}{D t}=\sum_{j=1}^{N} m_{j}\left(v_{i}^{\alpha}-v_{j}^{\alpha}\right) \frac{\partial W_{i j}}{\partial x_{i}^{\alpha}}$
$\frac{D v_{i}^{\alpha}}{D t}=\sum_{j=1}^{N} m_{j}\left(\frac{\sigma_{i}^{\alpha \beta}}{\rho_{i}^{2}}+\frac{\sigma_{j}^{\alpha \beta}}{\rho_{j}^{2}}\right) \frac{\partial W_{i j}}{\partial x_{i}^{\beta}}+f^{\alpha}$

Similarly, the SPH approximation of the shear strain $\operatorname{rate}\left(\varepsilon_{i}^{\alpha \beta}\right)$ in the water model, and the strain rate tensor $\left(\dot{\varepsilon}_{i}^{\alpha \beta}\right)$ and the rotation rate tensor $\left(\omega_{i}^{\alpha \beta}\right)$ in the soil model can be derived as follows:

$$
\begin{aligned}
\varepsilon_{i}^{\alpha \beta}=\sum_{j=1}^{N} & \frac{m_{j}}{\rho_{j}}\left(v_{i}^{\alpha}-v_{j}^{\alpha}\right) \frac{\partial W_{i j}}{\partial x_{i}^{\beta}} \\
& +\sum_{j=1}^{N} \frac{m_{j}}{\rho_{j}}\left(v_{i}^{\beta}-v_{j}^{\beta}\right) \frac{\partial W_{i j}}{\partial x_{i}^{\alpha}} \\
& -\left(\frac{2}{3} \sum_{j=1}^{N} \frac{m_{j}}{\rho_{j}}\left(v_{i}^{\gamma}-v_{j}^{\gamma}\right) \frac{\partial W_{i j}}{\partial x_{i}^{\gamma}}\right) \delta^{\alpha \beta}
\end{aligned}
$$




$$
\begin{array}{r}
\dot{\varepsilon}_{i}^{\alpha \beta}=\frac{1}{2} \sum_{j=1}^{N}\left(\frac{m_{j}}{\rho_{j}}\left(v_{i}^{\alpha}-v_{j}^{\alpha}\right) \frac{\partial W_{i j}}{\partial x_{i}^{\beta}}\right. \\
\left.+\frac{m_{j}}{\rho_{j}}\left(v_{i}^{\beta}-v_{j}^{\beta}\right) \frac{\partial W_{i j}}{\partial x_{i}^{\alpha}}\right) \\
\omega_{i}^{\alpha \beta}=\frac{1}{2} \sum_{j=1}^{N}\left(\frac{m_{j}}{\rho_{j}}\left(v_{i}^{\alpha}-v_{j}^{\alpha}\right) \frac{\partial W_{i j}}{\partial x_{i}^{\beta}}\right. \\
\left.-\frac{m_{j}}{\rho_{j}}\left(v_{i}^{\beta}-v_{j}^{\beta}\right) \frac{\partial W_{i j}}{\partial x_{i}^{\alpha}}\right)
\end{array}
$$

\section{SOIL-WATER INTERACTION MODELING}

When groundwater is seeping through the pores of a soil, viscous friction will produce drag on soil particles in the direction of water flow, so-called seepage force. This seepage force acts on the soil particles in addition to the gravitational force, and will be introduced into the momentum equations for soil and water as an external force according to the following model equation based on the Darcy's law:

$f_{\text {seepage }}=\gamma_{w} n \frac{\left(v_{\text {water }}-v_{\text {soil }}\right)}{k}$

where $\gamma_{\mathrm{w}}$ is the unit weight of water; $\mathrm{n}$ is the porosity; and $k$ is the soil permeability.

As saturated soil consists of soil and water mixed together while standard SPH models only handle one phase problem, it is necessary to develop a saturated soil model using in SPH simulation. This saturated soil model will be described as follows:

We assumed that the saturated soil domain in SPH can be divided into two separate phases, which are water phase and soil phase. The motion of SPH particles on each phase is solved separately using its own SPH governing equations, which are $\mathrm{SPH}$ for soil and SPH for water. These two-phases are then superimposed and the interaction between twophases will be taken into account through the seepage force, which is introduced into the momentum equation as mentioned before. In addition, the water pressure is also allowed to contribute to the soil pressure during the overlapping procedure. This allows us to simulate the pore water pressure, which always exists in natural saturated soil.

Accordingly, the momentum equations for saturated soil can be summarized as follows,

Momentum equation for soil phase

$$
\begin{aligned}
\frac{D v_{i}^{\alpha}}{D t}=\sum_{j=1}^{N} m_{j} & \left(\frac{s_{i}^{\alpha \beta}}{\rho_{i}^{2}}+\frac{s_{j}^{\alpha \beta}}{\rho_{j}^{2}}\right) \frac{\partial W_{i j}}{\partial x_{i}^{\alpha}} \\
& -\sum_{j=1}^{N} m_{j}\left(\frac{P_{i}}{\rho_{i}^{2}}+\frac{P_{j}}{\rho_{j}^{2}}\right) \frac{\partial W_{i j}}{\partial x_{i}^{\alpha}} \\
& -\sum_{a=1}^{N} m_{a} \frac{P_{a}}{\rho_{i} \rho_{a}} \frac{\partial W_{i a}}{\partial x_{i}^{\alpha}} \\
& +\sum_{a=1}^{N} m_{a} \frac{f_{i a}^{\text {seepage }}}{\rho_{i} \rho_{a}} W_{i a}+f_{i}^{\alpha}
\end{aligned}
$$

Momentum equation for Water phase

$$
\begin{aligned}
\frac{D v_{a}^{\alpha}}{D t}=\sum_{b=1}^{N} m_{b} & \left(\frac{\tau_{a}^{\alpha \beta}}{\rho_{a}^{2}}+\frac{\tau_{b}^{\alpha \beta}}{\rho_{b}^{2}}\right) \frac{\partial W_{a b}}{\partial x_{a}^{\alpha}} \\
& -\sum_{b=1}^{N} m_{b}\left(\frac{P_{a}}{\rho_{a}^{2}}+\frac{P_{b}}{\rho_{b}^{2}}\right) \frac{\partial W_{a b}}{\partial x_{a}^{\alpha}} \\
& -\sum_{i=1}^{N} m_{i} \frac{f_{i a}^{\text {seepage }}}{\rho_{i} \rho_{a}} W_{i a}+f_{a}^{\alpha}
\end{aligned}
$$

where the subscripts $i$ and $j$ represent for soil particles while $a$ and $b$ are used for water particles. It is clear that these momentum equations of saturated soil are different from that of single phase, equation (21). The presence of the seepage force in equations (26), (27), and the contribution of pressure from water to soil in equation (27) make them possible to simulate the effect of seepage force and pore water pressure in the saturated soil model, as a result the interaction between soil and water could be simulated through SPH.

In order to damp out the unphysical stress fluctuation and to prevent shock waves and the penetration of particles through the boundaries, an artificial viscosity has been employed to the pressure term in the momentum equation. The most widely used type is proposed by Monaghan and Lattanzio (1985), [12], and specified as follows

$$
\pi_{i j}= \begin{cases}\frac{-\alpha \bar{c}_{i j} \phi_{i j}+\beta \phi_{i j}^{2}}{\bar{\rho}_{i j}} & v_{i j .} x_{i j}<0 \\ 0 & v_{i j} . x_{i j} \geq 0\end{cases}
$$

in which $\alpha$ and $\beta$ are constants and are taken $0.01,1.0$ respectively, and $c$ represent the speed of sound. As well as having beneficial effects, artificial viscosity can also introduce unwanted numerical de- 
fects in some cases, among which the excess dissipation and false shearing torque in rotating flows (Dalrymple, and Knio, 2001).

The momentum equations for saturated soil after introducing the artificial viscosity to the pressure term are:

Momentum equation for soil phase:

$$
\begin{aligned}
\frac{D v_{i}^{\alpha}}{D t}=\sum_{j=1}^{N} m_{j} & \left(\frac{s_{i}^{\alpha \beta}}{\rho_{i}^{2}}+\frac{s_{j}^{\alpha \beta}}{\rho_{j}^{2}}\right) \frac{\partial W_{i j}}{\partial x_{i}^{\alpha}} \\
& -\sum_{j=1}^{N} m_{j}\left(\frac{P_{i}}{\rho_{i}^{2}}+\frac{P_{j}}{\rho_{j}^{2}}+\pi_{i j}\right) \frac{\partial W_{i j}}{\partial x_{i}^{\alpha}}+f_{i}^{\alpha} \\
& -\sum_{a=1}^{N} m_{a} \frac{P_{a}}{\rho_{i} \rho_{a}} \frac{\partial W_{i a}}{\partial x_{i}^{\alpha}} \\
& +\sum_{a=1}^{N} m_{a} \frac{f_{i a}^{\text {seepage }}}{\rho_{i} \rho_{a}} W_{i a}
\end{aligned}
$$

Momentum equation for Water phase:

$$
\begin{aligned}
\frac{D v_{a}^{\alpha}}{D t}=\sum_{b=1}^{N} m_{b} & \left(\frac{\tau_{a}^{\alpha \beta}}{\rho_{a}^{2}}+\frac{\tau_{b}^{\alpha \beta}}{\rho_{b}^{2}}\right) \frac{\partial W_{a b}}{\partial x_{a}^{\alpha}} \\
& -\sum_{b=1}^{N} m_{b}\left(\frac{P_{a}}{\rho_{a}^{2}}+\frac{P_{b}}{\rho_{b}^{2}}+\pi_{i j}\right) \frac{\partial W_{a b}}{\partial x_{a}^{\alpha}} \\
& +f_{a}^{\alpha}-\sum_{i=1}^{N} m_{i} \frac{f_{i a}^{\text {seepage }}}{\rho_{i} \rho_{a}} W_{i a}
\end{aligned}
$$

\section{BOUNDARY CONDITIONS}

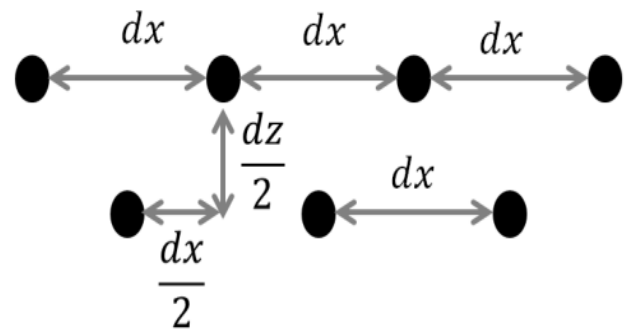

Figure 2. Arrangement of boundary particles

In this study, a dynamic boundary condition is used to represent the boundary particles, which are forced to follow the governing equations (continuity, momentum and state equations), but they are fixed. When the particles move closer towards the boundary, the density of the boundary particles increases, according to the continuity equation, which leads to an increase in the pressure following the equation of state. Subsequently, the force exerted on the approaching particles increases because of the pressure term in the momentum equation by the generations of repulsion between the material particles and the boundary particles (Dalrymple and Knio, 2001). The boundary particles are set in a staggered manner in order to prevent the particle leakage as shown in figure 2 .

\section{SCOUR OF THE GROUND BEHIND THE WEIR DUE TO OVERFLOW}

\subsection{Model description}

In order to evaluate our SPH model for soil-water interaction, simulations of the local scour behind the weir due to overflow have been carried out in this study.

The experiment done by Maeda et al. 2016, figure 3 shows a schematic sketch of the experimental setup. The experiment channel was $2.0 \mathrm{~m}$ in length, $0.3 \mathrm{~m}$ in width, and $0.3 \mathrm{~m}$ in height. In the tank, sedimentary soil $(0.5 \mathrm{~m}$ long, $0.3 \mathrm{~m}$ wide, and $0.1 \mathrm{~m}$ high) was installed as the ground, the starting point of which was $1.0 \mathrm{~m}$ from the upstream end. For the fluid force, a circulation flow was generated using a submersible pump to unify the external force.

The experiment was recorded by using a highspeed camera (200-400 frame/Sec) and a video camera $(29.97$ frame/Sec) to observe scouring and corrosion.

In this experiment, a weir with $h_{\text {weir }}=80 \mathrm{~mm}$ in height was installed at the uppermost side of the ground, and a water sealing plate was set at the lowermost side of the channel to control the water level in the downstream. The Geo-material in this experiment was Toyoura sand $\left(D_{50}=4.73 \mathrm{~mm}\right)$. The initial water-level difference expressed as $\Delta h$ and $\Delta h$ was $20 \mathrm{~mm}$.

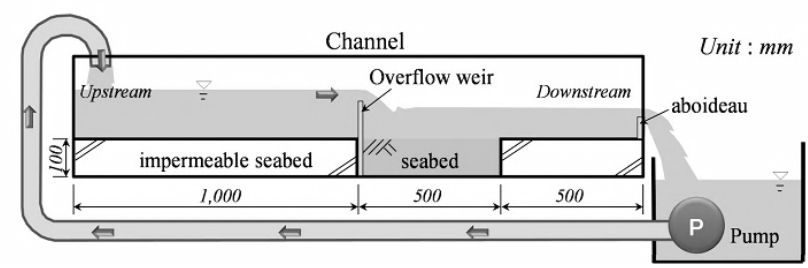

Figure 3. Schematic sketch of the experiment, Matsuda 2013

\subsection{Numerical simulations}

The simulation of the local scour was carried out using the proposed SPH model.

The dry soil is modeled by one type of particles with uniform material properties. These particles have the following properties: Young's modulus $E$ $=150 \mathrm{MPa}$ and Poisson's ratio $v=0.3$. The water particles have density of $\rho=1.0 \mathrm{~g} / \mathrm{cm} 3$ and viscosity $\mu=10^{-3} \mathrm{Ns} / \mathrm{m}^{2}$. 
In total 12801 and 25602 particles representing the soil and water particles, respectively, with an initial distance $0.002 \mathrm{~m}$ was used.

\subsection{Results and analysis}

To validate the model, the computed free surfaces and bed evolution profiles using the SPH model are compared with the experimental data (Maeda et al. 2016) in figure 4 at two different time instants before and after formatting the scouring, i.e. $\mathrm{t}=0.5$ min, and $2.0 \mathrm{~min}$; respectively.

The saturated soil consisted of two-phases, water and soil, and each phase have to handle separately using its own SPH model in order to obtain the field variables of each particle. After the field variables of particles in each phase are computed, the interaction between soil and water can perform. The water pressure is permitted to contribute to the pressure of soil particles through the SPH summation, and thus the pore water pressure can be simulated. The seepage force is also computed as a function of the relative velocity between soil and water particles, and be- cause of the presence of the seepage force in the momentum equations (18), (19), these water particles will force soil particles to move together, so the saturated soil mixture is effectively incompressible.

The comparisons in figure 3 indicated that the SPH computed free surface profiles match the measured interface-contours quite well.

The development of the scour and the profile of the bed level, is reproduced in a characteristic manner by the numerical model. However, the shape of soil erosion is slightly different compared with the experiment. Also, as can be seen from figure 2, the fluid particles are able to enter the sediment layer up to a certain depth, which is similar to seepage. The exerted forces by the fluid particles in the pores may be interpreted as a mix of pore water pressure and seepage force forces. The overall agreement is nonetheless satisfactory, a more accurate constitutive model for soil, not yet implemented in this research, would take into account the plastic strain in the plastic flow regime.

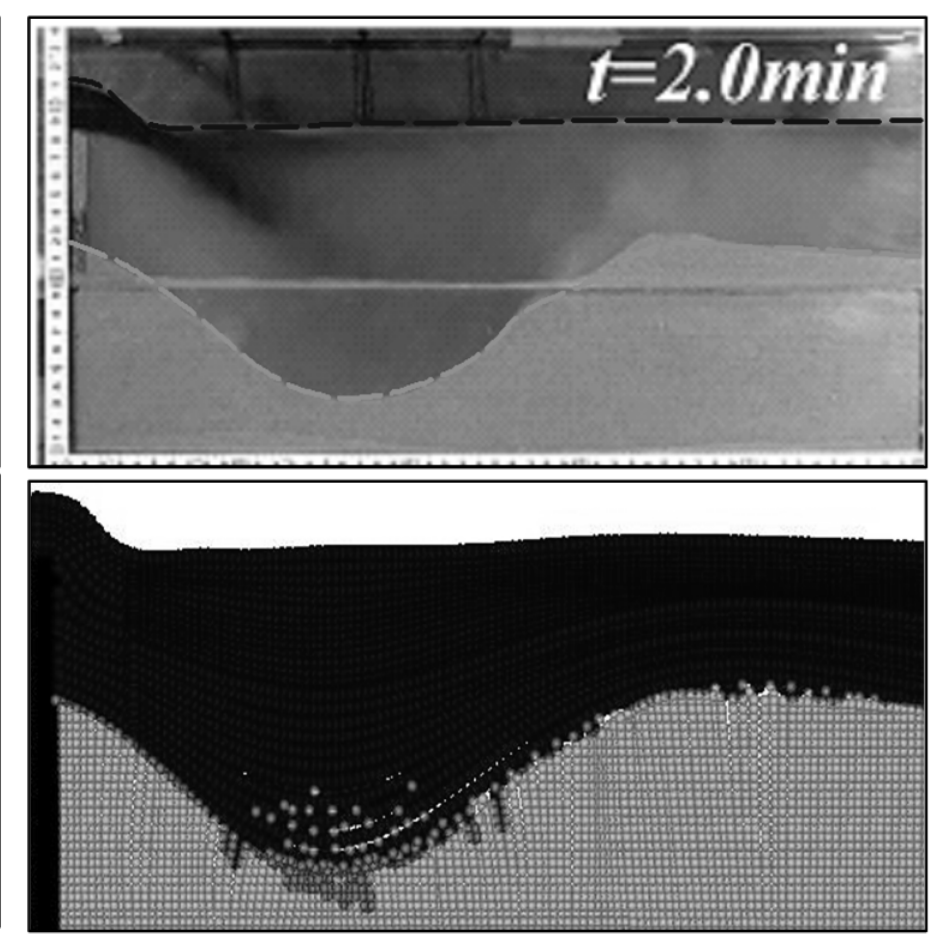

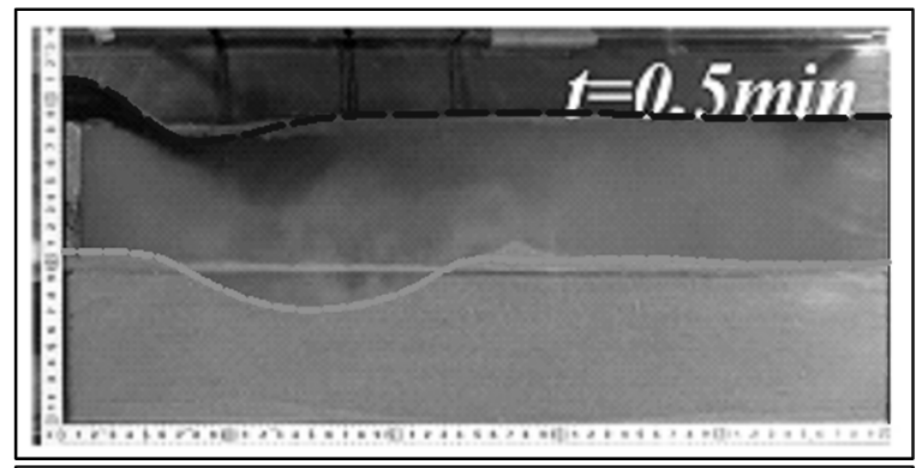
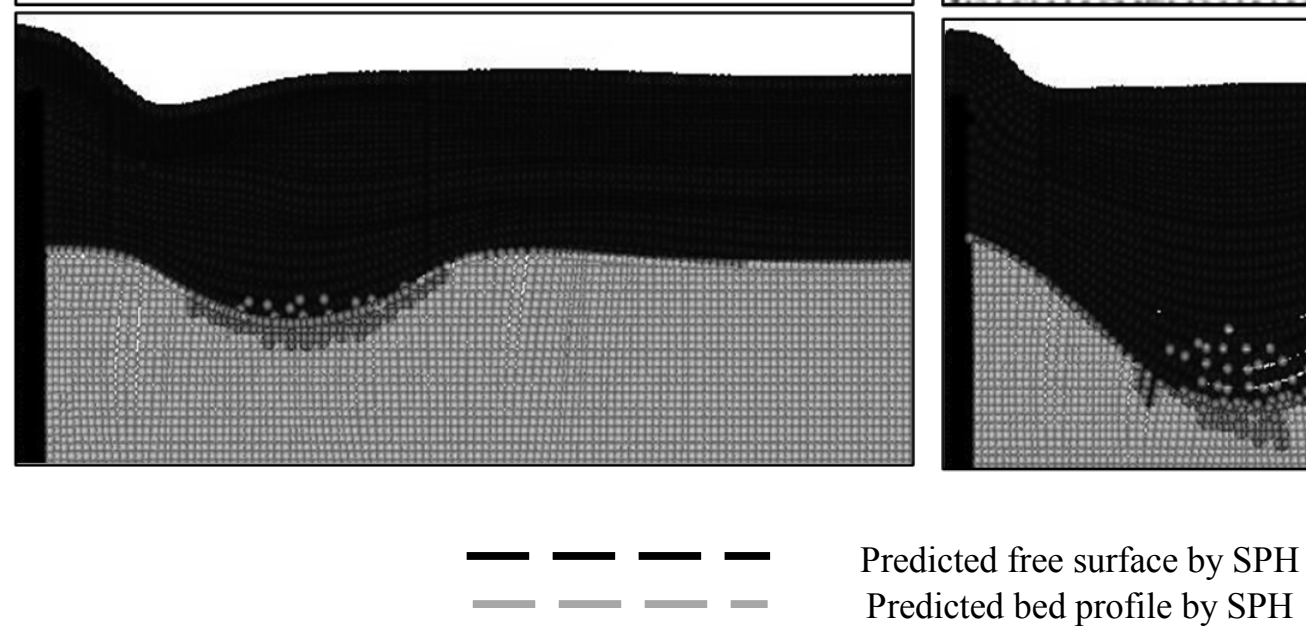

Figure 4. Snapshots of the local scour experiments ( $\mathrm{t}=0.5,2.0 \mathrm{~min})$ compared with SPH simulations 


\section{CONCLUSIONS}

The development of an improved smoothed particle hydrodynamics to simulate the behavior of soilwater interaction has been described through this paper. Water was modeled as a viscous fluid with week compressibility while the soil was modeled as an elastic-perfectly plastic material. Interaction between soil and water was modeled by means of pore water pressure and seepage force. Simulation of the Louvain erosional dam-break experiment presented has been presented. The results have shown that the extremely large deformation and failure of soil can be handled in SPH without any difficulties.

\section{ACKNOWLEDGMENT}

This work was supported by the Ministry of Education, Culture, Sports, Science, and Technology, Japan (MEXT) and JSPS KAKENHI Grant Number $15 \mathrm{~F} 15369$.

\section{REFERENCES}

Abdelrazek, A. M., Kimura, I. \& Shimizu, Y. 2014. Comparison between SPH and MPS Methods for Numerical Simulations of Free Surface Flow Problems. Journal of Japan Society of Civil Engineers. , Ser.B1 (Hydraulic Engineering), 70 (4): I 67-I 72.

Abdelrazek, A. M., Kimura, I. \& Shimizu, Y. 2014. Numerical Simulation of Snow Avalanches as a Bingham Fluid Flow Using SPH method. Proceedings of river flow 2014, 7th international conference on fluvial hydraulics, Lausanne, Switzerland, 3-5 Sept 2014, 1581-1587, CRC Press, Taylor \& Francis Group .

Abdelrazek, A. M., Kimura, I. \& Shimizu, Y. 2014. Numerical simulation of a small-scale snow avalanche tests using nonNewtonian SPH model. Journal of Japan Society of Civil Engineers, Ser. A2 (Applied Mechanics (AM)), 70 (2): I 681-I 690.

Abdelrazek, A. M., Kimura, I. \& Shimizu, Y. 2015. Numerical Simulation of Granular Flow Past Simple Obstacles using the SPH Method. Journal of Japan Society of Civil Engineers, Ser. B1 (Hydraulic Engineering), 71 (4), I_199I_204.

Abdelrazek, A. M., Kimura, I. \& Shimizu, Y. 2016. Simulation of three-dimensional rapid free-surface granular flow past different types of obstructions using the SPH method. Journal of Glaciology, (in press).

Bui, Ha H., Sako, K. \& Fukagawa, R. 2007 . Numerical simulation of soil-water interaction using smoothed particle hydrodynamics (SPH) method. Journal of Terramechanics, 44, 339-346.

Bui, Ha H., Fukagawa, R., Sako, K. \& WELLS, J. C. 2010. Slope stability analysis and discontinuous slope failure simulation by elasto-plastic smoothed particle hydrodynamics (SPH). Géotechnique, 61 (7), $565-574$.

Dalrymple R.A. \& Knio O. 2001. SPH modelling of water waves. Proc. Coastal Dynamics: pp. Sweden.779-787.

Fraccarollo, \& Capart H. 2002. Riemann wave description of erosional dam-break flows. Journal of Fluid Mechanics, 461:183-228.
Liu G.R. \& Liu M.B. 2003. Smoothed particle hydrodynamics: a meshfree particle method. World Scientific.

Liu G.R. \& Liu M.B. 2010. Smoothed Particle Hydrodynamics (SPH): an Overviewand Recent Developments, Arch Comput Methods Eng 17: 25-76.

Maeda1, K., Matsuda, T., \& Saito H. 2016. Instability on dike with scour due to surface flow and seepage. The 15th Asian Regional Conference on Soil Mechanics and Geotechnical Engineering, 2 (29): 1065- 1068.

Matsuda, T. 2013. A study on the tsunami disaster mechanism on coastal structures due to instability of rubble mound and seabed ground and its countermeasure. Doctoral Thesis.

Monaghan J.J., \& Lattanzio J. C. 1985. A refined particle method for astrophysical problems. Astron Astrophys., 149:135.

Monaghan, J. J. 1994. Simulating free surface flows with SPH. J. Comput. Phys., 110: 399-406

Monaghan, J. J. \& Kocharyan, A. 1995. SPH simulation of multiphase flow. Comput. Phys. commun., 87, 225-235.

Shinichi, Y. \& Toshihiko, U. 2011. Simulation of Granular Flows and Pile Formation in a Flat-Bottomed Hopper and Bin, and Experimental Verification. Materials , 4(8): 1440 $-1468$.

Yaidel, R.L., Dirk, R. \& Carlos, R.M. 2013. Dynamic particle refinement in SPH: application to free surface flow and non-cohesive soil simulations. Computational Mechanics, $51,731-741$ 